\title{
Implementing tools to meet the Floods Directive requirements: a "procedure" to collect, store and manage damage data in the aftermath of flood events
}

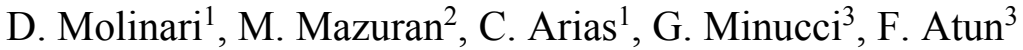 \\ \& D. Ardagna ${ }^{2}$ \\ ${ }^{1}$ Department of Civil and Environmental Engineering, \\ Politecnico di Milano, Italy \\ ${ }^{2}$ Department of Electronics, Information and Bioengineering, \\ Politecnico di Milano, Italy \\ ${ }^{3}$ Department of Architecture and Urban Studies, \\ Politecnico di Milano, Italy
}

\begin{abstract}
The aim of this paper is to present a "procedure" to collect and store damage data in the aftermath of flood events. The activity is performed within the Poli_RISPOSTA project (stRumentI per la protezione civile a Supporto delle POpolazioni nel poST Alluvione), an internal project of Politecnico di Milano whose aim is to supply tools supporting Civil Protection Authorities in dealing with flood emergency. Specifically, the aim of this paper is to discuss the present implementation of the project, highlighting challenges for data collection, storage, analysis and visualisation. Data can have different formats (e.g. paper based vs. digital form, different digital files extensions), refer to different aspects of the phenomenon (i.e. hazard, exposure, vulnerability and damage), refer to different spatial and temporal scales (e.g. micro vs. meso scale, different phases of the flood event) and come from different sources (e.g. local authorities, field surveys, crowdsourcing). Therefore a multidisciplinary approach which includes expertise from ICT, geomatics, engineering, urban planning, economy, etc. is required. This paper first describes a conceptual map of the issue at stake, then it discusses the state of the art of the implementation, taken as reference the Umbria flood in November 2012. Impacts of the project are discussed with
\end{abstract}


particular attention to their utility to meet some of the "Floods Directive" (Directive 2007/60/EU) requirements: (i) to create a reliable and consistent database; the latter should be the basis on which damage models can be defined/validated and thus risk can be mapped; (ii) to supply a comprehensive scenario of flood impacts according to which priorities can be identified during the emergency and recovery phase.

Keywords: flood risk management, flood damages, disaster databases, flood damage maps.

\section{Introduction}

In recent years, awareness of a need for more effective disaster data collection, storage, and sharing of analyses has developed in many parts of the world, also in the wake of several policies that, at different levels of government, implicitly or explicitly required to face the problem at stake (e.g. the Hyogo framework for Action [1], the EU disaster prevention framework [2], the European Union Solidarity Fund [3], the Green Paper on Insurance of Natural and Man-made Disasters [4]).

Among natural disasters, this paper focuses on floods. Having more reliable data on flood impacts is of paramount importance for improving pre and post event risk reduction strategies. For instance De Groeve et al. [5] suggest three application areas for (flood) loss data: loss accounting, disaster forensics and risk modelling. In the aftermath of flood events, the principal motivation for recording the impacts of floods is loss accounting. This information is crucial at different levels of governance/risk management. At the local level, civil protection and policy makers (i.e. mayors) need loss accounting in order to identify priorities for the emergency and the recovery-reconstruction phases while insurers use this information to compensate victims. At the subnational/national level, loss accounting is required by policy makers for fund allocation, for addressing damage compensation and recovery. At the international level the interest is on financial and humanitarian aid.

In peace time, flood loss data are required to improve knowledge of the mechanisms leading to flood impacts; to analyse the causes of disasters through measuring relative contribution of hazard, exposure, vulnerability and coping capacity (i.e. the response to the flood). This is what is called disaster forensic. The objective of disaster forensic is twofold: (i) to enhance disaster management from lessons learnt, and (ii) to improve risk mitigation strategies by increasing the capacity of modelling and forecasting flood damage.

Within this context, this paper presents the Poli-RISPOSTA project (stRumentI per la protezione civile a Supporto delle POpolazioni nel poST Alluvione), an internal project of Politecnico di Milano supporting interdisciplinary research with a direct impact on the society. The main intention of Poli-RISPOSTA is to build with and for the Civil Protection (CP) a model, tools and advanced technical solutions for collecting, mapping and evaluating post-flood damage data. In fact, as the consequence of the policies discussed above, the need for enhanced methods and procedures for post-event damage 
assessment has been increasingly demanded also by Italian local authorities. Moreover, requirements imposed by the European "Floods" Directive 2007/60/EC play a major role for flood risk.

In order to protect people and assets from the impact and consequences of floods, the EU Floods Directive requires that flood risk management plans (FRMPs) will be based not only on various flood hazard scenarios but also on risk assessments which must present the potential adverse consequences of floods for "human health, the environment, cultural heritage, and economic activity." There is then an increasing need to obtain more reliable, high quality flood impact data that can serve numerous purposes:

(i) to create a reliable and consistent database; the latter should be the basis on which damage models can be defined/validated and thus risk can be mapped. Flood risk maps are, in their turn, the main tools on which FRMPs are defined.

(ii) to supply a comprehensive scenario of the consequences of floods according to which priorities can be identified during the emergency and the recovery phases.

Poli-RISPOSTA wants to address these needs.

In order to provide appropriate solutions, one of the key principles of the project is working with stakeholders, not for them. Stakeholders involvement has been increasingly demanded by both European policies on risk mitigation (i.e. the quoted EU Floods Directive is emblematic for the problem at stake) and research project calls at both national and international levels (see e.g. the FP7 program) but there is a significant difference between interviewing stakeholders to obtain feedback on work that has already been carried out in research centres and developing tools and methods jointly. The last modus operandi is, in authors' opinion, the only way to get efficient and feasible solutions to the problem at stake. Accordingly, stakeholders will be actively involved during the entire project by means of meeting, participatory activities, exercises, etc.

Last but not least, it is worth noticing that Poli-RISPOSTA is an interdisciplinary project where experts from several fields are involved as ICT, geomatics, engineering, urban planning, economy, etc.

The conceptualisation of the problem addressed, the approach followed in the project as well as challenges of Poli-RISPOSTA (also in terms of required expertise) are discussed more in detail in section 2. Section 3 describes the current state of the project: challenges regarding data acquisition and requirements that are needed to gather data before, during and after a flood are identified. Section 4 concerns the steps required for the remaining part of project. The paper ends with conclusion including deductions coming from the paper.

\section{Problem conceptualisation}

The general objective of Poli-RISPOSTA can be identified in the development of a "complete" flood scenario describing both the physical features of the forcing event (i.e. the flood) as well as its impacts and the capacity of societies to face them. In order to accomplish with risk mitigation objectives (e.g. those imposed 
by the EU Floods Directive) such a scenario must be developed both ex-ante and ex-post. Before an event occurs "complete" scenarios provide a picture of the most risky areas and allow identifying strategies to mitigate risk and to cope with hazardous events. After the event occurrence, the objective is instead to figure out real impacts and to identify priorities for the emergency and the recovery phase (fig. 1). A comparison of the ex-ante and ex-post scenarios allows finally to infer lessons towards an improvement of both the capacity of predicting the event (and its consequences) and to cope with it.

To achieve these goals, tools and advanced technical solutions to collect, store, analyse and represent a multitude of data must be developed within PoliRISPOSTA (fig. 1). After an event occurs, such data regard both physical effects of the forcing event (as flooded areas, water depth and velocity inside it, the occurrence and localisation of landslides, etc.) and observed damages on the different sectors of the society (i.e. people, economic, and human activities), the natural and built environment (i.e. residential and industrial buildings, infrastructures, public and cultural heritage, ecosystems). Damages can be due to the physical contact of the flooding water (i.e. direct damages) or induced by the first (i.e. indirect damages); both (ii) tangible (i.e. monetary) and intangible data must be taken into account. Moreover, data on mitigation actions implemented by emergency services and lay people before and during the flood is of interest as these actions influence both physical effects and damages.

Before the event, data regard instead results from hazard, exposure and vulnerability modelling. Also in this case, information must be managed with respect to the different variables characterising the physical scenario as well as required to estimate risk on the different items potentially affected by the floods.

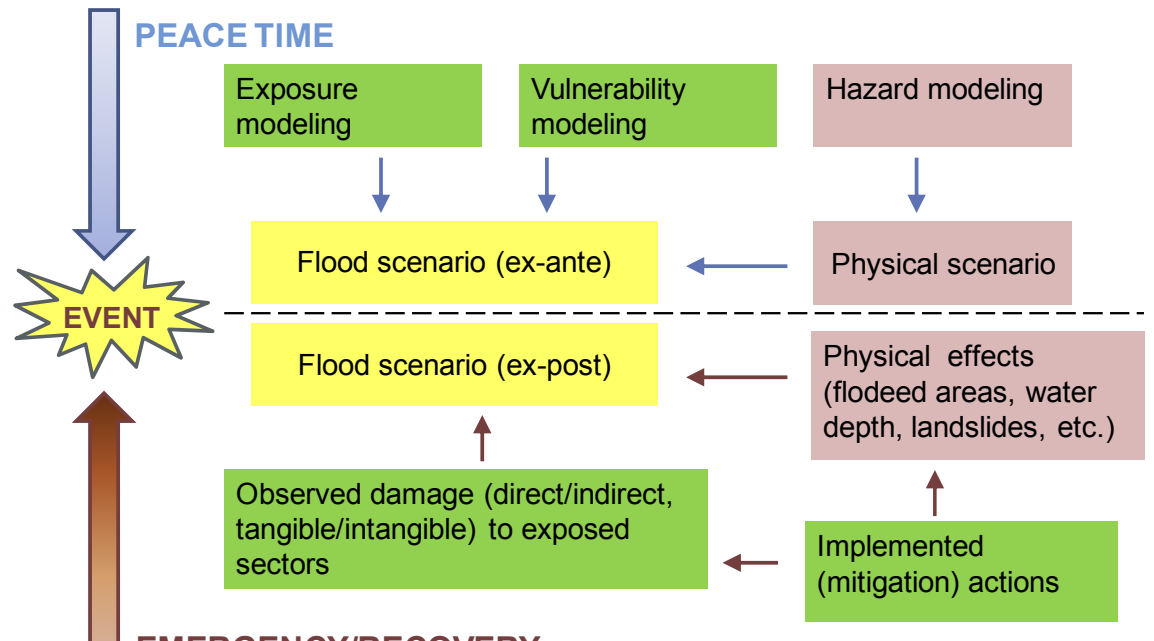

Figure 1: Objective of Poli-RISPOSTA and data of interest. 
Poli-RISPOSTA objectives can be achieved by developing of an Information System (IS) to coordinate and support all the activities earlier described. With respect to this, a cyclic process is followed in the project (see fig. 2). First, features of data to be managed and required elaborations to develop complete scenarios are analysed in order to identify critical aspects to be managed by the IS; such criticalities are translated into requirements for the IS. The next step is the design and the development of a prototype which keeps into account identified requirements. The application of the prototype to a real case allows to update data analysis and corresponding IS requirements; according to this the prototype is revised for new applications and tests. In this way, the final product will be created based on step-by-step refinements, also according to new data and analyses which could be available/required after the first development of the IS.

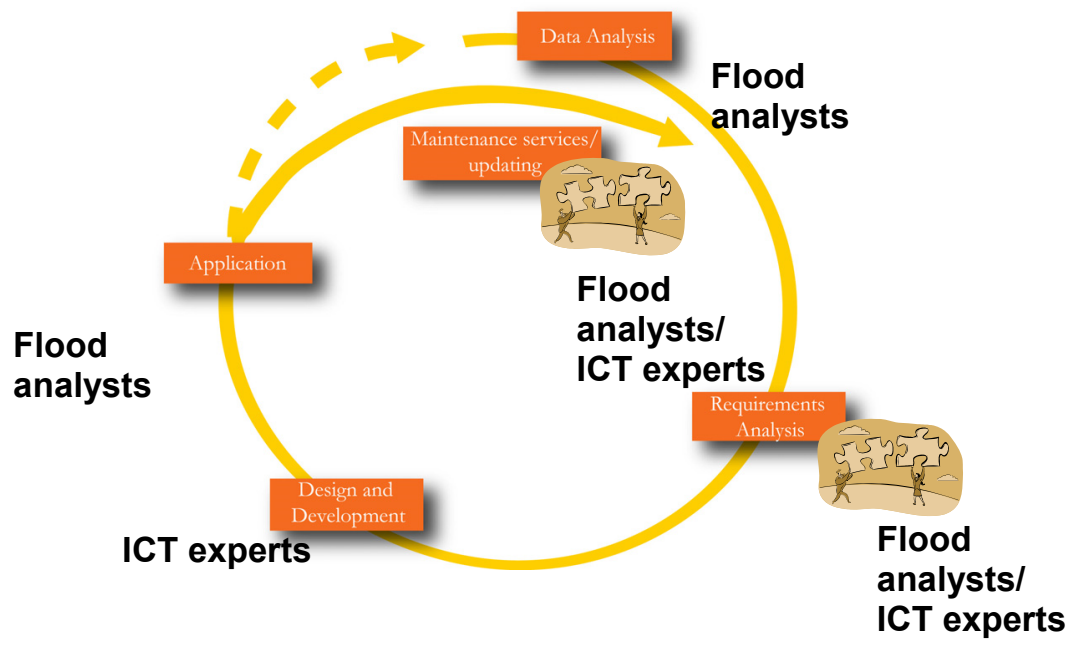

Figure 2: The cyclic process adopted in the Poli-RISPOSTA.

The complexity of the problem at stake implies several challenges for PoliRISPOSTA, with respect to the current state of the art. In the following the most relevant are discussed.

First, tools for systematic loss accounting are not very well developed. The way in which flood damage data are presently collected and stored implies several problems for an efficient, multipurpose use of data as wished in [5] and [6]. The main problem of existing disaster databases concerns data comparison and management. This is due to a lack of agreed standard to collect and store damage data. Specifically, several differences can be found in existing databases regarding:

- recorded losses. This depends on: (i) the intent of the reporting activity (i.e. insurance companies, governmental agencies and NGOs collect data for different purposes; for this reason, flood loss records are often not representatives of the real impact of floods as they focus only on certain items 
at risk and/or types of damage), (ii) the time of reporting, and (iii) present capacity of estimating all types of damages (e.g. indirect or secondary damages are not so evident in the aftermath of an event and are difficult to evaluate in monetary terms).

- The scales of reporting. Flood loss data can be recorded at different spatial and temporal scales, according to the intent of the report and to who is leading the reporting activity (i.e. their role and responsibility). However, aggregating/disaggregating damage over space and time is not straightforward.

- The economic rationale. There are different methods to evaluate monetary loss, e.g. taking into account inflation (i.e. depreciated value), purchasing parity (i.e. replacement value), insured losses, etc.

One of the main challenges Poli-RISPOSTA has to face is to develop tools for the survey and collection of flood loss data that overcome the above limits, guaranteeing high quality, consistent and reliable data, in the philosophy that "the quality of disaster databases can only be as good as the reporting system" [7]. Contrary to common practice, Poli-RISPOSTA wants to work at the local level in order to meet two basic requirements of flood loss data: (i) going into details of phenomena/aspects leading to damage and (ii) reporting all the events, including small ones (like multi spot flash floods in mountain regions) which are presently discounted by national/international databases [8][9]. Data at upper levels, for strategic and policy making purpose, can be obtained in a second step by proper aggregation rules. On the other hand, Poli-RISPOSTA wants to provide a "complete picture" of a disaster, identifying damage to various sectors of the economy and society. From this perspective, the PDNA - Post Disaster Needs Assessment methodology resulting from the collaboration of a number of institutions, including the EU Commission, United Nations, the World Bank and others is a very important example (for an application see [10]).

Linked to the previous point is the development of technological solutions for data acquisition. Indeed, while damage data at the meso or macro scale can be inferred from indirect sources (e.g. public accounting, researches, newspapers, and regulations), local data are often collected by means of field surveys. Tools should then be developed in order to support data survey in digital format. Such tools should provide real time data storage (in a database) and their visualisation in terms of maps, supporting this way the field survey/emergency phase (e.g. supporting the coordination of survey team). With respect to this, the DARMsys developed by the Queensland Reconstruction Authority in Australia can be taken as reference [11].

The need of managing collected data also in terms of visualisation and spatial analysis represents another challenge for Poli-RISPOSTA. Since there is not a standardized way to collect spatial data in the case of floods, data collected in the aftermath of flood events are commonly in different formats that make data not immediately usable for spatial analysis. This is not the case, e.g., in ex-ante risk assessments where data of interest are directly produced to be handled by GIS tools [12]. Creating flood databases is common practice, but not always in GIS standard compatible formats. This point must be addressed by Poli- 
RISPOSTA, providing tools for data storage which also satisfy requirements of data visualisation and spatial analysis.

Last but not least, as any technological problem, an interdisciplinary approach is required by the project that put together computer scientists and domain experts.

With respect to this, the further challenge of Poli-RISPOSTA is that not only domain experts come from different disciplines but also computer scientists are heterogeneous. As regard domain experts, the need of analysing both the physical feature of the event as well as its consequences (both in monetary terms and with respect to intangible damages) implies expertise from engineering, urban planning, sociology, economy, etc. With respect to computer sciences ICT experts are required for the collection, storage and management of data of interest. Moreover, the need of representing data in terms of maps as well as of carrying out spatial analyses (see section 2) requires involving experts from geomatics.

\section{Present implementation of Poli-RISPOSTA}

The project is currently evolving towards the third step of the cycle in fig. 2. A significant effort has already been put into the first two steps that represent a crucial part of the cycle since their outputs are the starting point for the development of the IS. Therefore, a good analysis both of the domain and of the requirements is fundamental. Particularly at this stage of the project, the interdisciplinary approach discussed above is needed to combine the two areas of expertise; flood analysts and ICT experts have to collaborate side by side to define the most complete picture of the application scenario. The better the result is, the better the basis for the implementation of the IS will be. Indeed, during the lifetime of the IS, new needs and requirements might come up that need to be incorporated into the system. Therefore, we need an agile approach where the feedbacks gathered from the running system are used to improve and refine its specifications (i.e. the cyclic approach shown in fig. 2).

In the next two subsections the results of the first two steps are discussed in details. Results highlight the complexity of the problem at stake.

\subsection{Data analysis}

According to the cyclic process described in fig. 2, the first step in designing the IS consists of an analysis concerning both data characteristics and types of required elaboration to be performed. This analysis was carried out on the basis of the flood event occurred in the Umbria Region - Central Italy in November 2012 [13]. On that occasion the regional CP asked Politecnico di Milano to develop a report (under construction) describing the event and its consequences at the regional level. Researchers activity focused on two aspects: (i) the development of an ex-post scenario to help CP to figure out event impacts [14], to identify priorities for recovery and reconstruction and to verify effectiveness of emergency plans; (ii) the development of an ex-ante scenario to be compared 
with the first to verify whether or not existing risk assessment and mitigation strategies are suitable to deal with flood risk in Umbria. Indeed, results from this experience are the starting point of Poli-RISPOSTA, allowing both to recognise needs and requirements in terms of data analysis and elaboration for the scenario development and to analyse the features of (available or required) data on which such activity should be performed (see section 3.2).

With respect of data features, besides the fact that they refer to the different domains discussed in section 2 (i.e. risk modelling, observed physical effects and damage on the different sectors, mitigation actions), other important features were recognised which imply requirements for the IS (see section 3.2); availability in time is one of them. Data are available at different times. This is due, on the one hand, to the nature of the data itself; for example, modelling data are available before an event occurs, indirect damages (e.g. disruption of economic activities, of basic services to the population, the loss of rental income) are not evident in the aftermath of an event but some months later, etc. On the other hand, norms regulating damage compensations count. The latter identify which damages are refunded by law and which are the deadlines to ask for compensation; for this reason, both public and private subjects give priority to determine reimbursable damages while other types of losses are assessed in a second step (e.g. in Italy damage to infrastructures must be declared by regional authorities 20 days after the event while damage to residential buildings 90 days after). However, generally speaking, data of interest are available before the event or after the event, in a time window ranging from few days to 1 year.

Another important feature is the spatial scale; data can refer to individual objects (e.g. damage to a building, a bridge, a fabric), the local scale (e.g. number of evacuees in a municipality), the large scale (e.g. traffic disruption at the province, flood zones in the river basin) or to the regional /national/international scale (e.g. indirect damage to ecosystems). The Euclidian dimension is also linked to this feature and to the need of representing data (analyses) in terms of maps (see below). Data can be represented as points (e.g. the damage to a building), lines (e.g. length of damaged roads) or areas (e.g. flooded areas).

The source of data is another important aspect. Some data are acquired by means of field surveys (e.g. direct damage to buildings, water depth inside the flooded area) for which suitable tools should be designed (see sections 3 and 4). Other data are directly produced by the CP (e.g. flood forecasts); finally several data are recorded by other subjects (e.g. local authorities, service suppliers, research centres) and must be "simply" collected by the CP.

The present data format is an additional characteristic to be taken into account. Coming from different sources, data can have different formats: papery based or digital. In the second case, recognised formats are heterogeneous: features, texts, spreadsheets, images or multimedia.

Last but not least, not only quantitative (e.g. observed damage) but also qualitative data (e.g. vulnerability features, emergency actions) are of interest.

In order to reproduce the complete event scenarios, the experience in the Umbria region highlighted that data of interest not only are heterogeneous but 
should also be managed in several ways. Most common analyses regard aggregation/disaggregation in space and time (e.g. determine damage over a municipality or one months after the event), filtering (e.g. evaluate damage to a specific sector), visualisation (e.g. in terms of graphs, diagrams, maps) and spatial analysis (e.g. determine the water depth at certain location, compare damage occurring in different municipalities).

In the next section requirements for the IS linked to both data features and required elaborations are discussed.

\subsection{System requirements}

Data heterogeneity implies several challenges for the IS development. To manage data effectively (in order to produce those elaborations which are required to develop a complete event scenario), criticalities highlighted in section 3.1 were translated into system requirements, according to the cyclic process in fig. 2:

1. Temporal tracking and storage of data: some data are available before the event, some are gathered during, other are collected after it; thus the system should allow to store and manage data collected at different times. As some information might deteriorate quickly after a flood event (e.g. the level of water, the memories of people affected by the event, etc.), the system must recall users on data to be recorded at each time. Moreover, for some data the interest is in keeping their history over time (that is, the way they change over time), for others only their current (or most updated) status needs to be known. Criteria must be defined in this regard to be embedded in the IS.

2. Data aggregation/disaggregation: Data are gathered at different scales; an approach is required to identify rules according to which data must be aggregated/disaggregated to guarantee information coherence.

3. Data redundancy prevention: most of data come from different sources; this reflects in several issues. In fact, data are gathered in many different formats (spreadsheets, documents, audio, video, etc.) that provide information of invaluable importance that could also be repeated. The system should define criteria according to which data are stored or not (e.g. quality of data, time of acquisition, source reliability, etc.).

4. Data pre-processing: Data can come in several formats that are not necessarily compatible with their storage in a database or for spatial analysis. The IS can support only pre-defined format(s). Accordingly, procedures for data preprocessing (for users) must be defined. Likewise, it is important to support the process of structuring (when possible) and organizing that information that is semi-structured or unstructured (e.g. pictures, drawings, audio files and so on).

5. Data acquisition: Some data are collected by means of field surveys. A tool should be developed to support data survey in digital format (e.g. tablet). Other data must be simply collected from other sources; accordingly the IS must allow data acquisition from different sources/users (see the next point).

6. Multi-owners environment: Different users will use the IS in different way (e.g. to insert data, to analyse data, to visualise data elaboration). Possible users 
must be identified as well as allowed actions for each users. This implies to create different user permissions in the IS (also in remote).

7. Data management: The IS must support several data analysis: aggregation/disaggregation, visualisation, filtering, querying, etc. both during the collection/survey phase and at the end of this activity. Pre-defined tools for data analysis must be developed within the IS to facilitate/make quicker the scenarios development.

\section{Next steps}

Coherently with the cyclic process in fig. 2, next steps of Poli-RISPOSTA are towards the implementation of the IS; in particular, the first milestone concerns the development of a first IS prototype. The design and implementation of the prototype will be performed in close collaboration with the $\mathrm{CP}$ by means of participatory processes, exercises, etc. according to the project philosophy which considers the involvement of stakeholders as key to get efficient and feasible solutions.

Next efforts of Poli-RISPOSTA can be grouped specifically into three main activities which are all required to develop the IS:

1. Database design and development. The IS is supported by a database where all the collected data are stored. Therefore, it is important to define its structure in order to be able to manage all the data introduced so far in a flexible way, so to support future changes or updates. The database must keep into account all the features of the data and the kind of manipulations to be performed on them, thus, it is designed according to the data and requirements analysis introduced in Section 3. Particular effort will be put into the depiction and management of geographical data that play a key role in the project.

The first version of the database will be designed and developed according to the data available from the 2012 Umbria flood, however, future events will be used to enrich data and requirement analysis and as a consequence to improve the design and development of the database itself.

2. Providing the software for data management. According to data requirements identified in section 3.2, software tools are needed with a twofold aim. On the one hand, tools for data acquisition will be developed. Such tools will support both the direct survey of flood damage data on the field and the collection of data from other sources by the CP. In both cases the software should embed/match with tools and procedures presently adopted by $\mathrm{CP}$ to perform data collection. On the other hand, tools for the reconstruction of the flood scenario must be provided. This means to supply the software required to analyse, interpret and represent collected data. It is worth noticing that the two "objectives" are not disconnected. In fact, a first reconstruction of the scenario is required in the first hours after the event to make available the identification, even with limited precision, of the flooded areas and affected items. This information will be used during the collection/field survey of data to identify and track investigated items. 
This activity will be performed taken as reference data elaboration produced/required after the November, 2012 flood in Umbria. At the same time the structure of the DB developed during activity 1 will be carefully taken into consideration. Actually, the development of the DB and of the software must be considered as complementary activities, requiring continuous interaction and feedbacks. Accordingly, the two activities should be done in parallel rather than one after the other.

3. Definition of a procedure to carry out data collection and elaboration. The last activity consists of the definition of a procedure to help practitioners to use the IS, in terms of both data collection and analyses. With respect to the first point, guidelines will be produced specifying which data should be collected, when, at which scale, by whom, in which format, etc. Regarding the second aspect, a framework will be drawn up detailing steps, data analyses and elaborations required to produce a complete event scenario. The objective is to create a procedure to be adopted by $\mathrm{CP}$ as a standard in case of flood, to make easier and quicker the analysis of the event. Also this last activity is strictly interconnected with the others and must be carried out in parallel with them.

The prototype that will be produced at the end of the three activities will be tested during a CP exercise in the Umbria region in autumn 2014. The test will allow to possibly modify IS requirements (and corresponding features) as described in the iterative process in fig. 2. The evolution of the IS according to test results will be the objective of next research efforts.

\section{Conclusion}

The objective of this paper is to present the Poli-RISPOSTA project, an interdisciplinary project of Politecnico di Milano providing novel and enhanced methods and procedures for post-flood damage assessment. The latter are a key prerequisite for improving pre and post event risk reduction strategies as required (among the others) also by the EU Floods Directive. Having more reliable flood loss data is of paramount importance for loss accounting, disaster forensic and risk modelling.

Efficient solutions imply the use of advanced technological tools; for this reason an interdisciplinary approach is required that put together expert's domain (i.e. flood analysts) and computer scientists.

By describing the current level of implementation of the project, the paper wants to highlight two peculiarities of the problem at stake. On the one hand, its complexity both in terms of methodological gaps, data to be handled, elaborations to be performed and the variety of expertise which is required. On the other hand, the need to work with stakeholders (i.e. the users of developed tools) to get feasible and effective solutions.

\section{Acknowledgements}

The authors acknowledge all the people involved in the Poli-RISPOSTA project for their useful feedback on the paper. Authors also acknowledge with gratitude 
the Umbria Region Civil Protection authority (and its staff), which strongly encourages/actively takes part in this research.

\section{References}

[1] ISDR, Hyogo framework for Action 2005-2015: Building the resilience of nations and communities to disasters, http: \www.unisdr.org/wcdr, 2009.

[2] Council of the European Union, Council Conclusions on a Community framework on disaster prevention within EU. 2979 ${ }^{\text {th }}$ Justice and Home Affairs Council meeting. Brussels, 30 November 2009.

[3] Council Regulation (EC) No 2012/2002 of 11 November 2002 establishing the European Union Solidarity Fund.

[4] EC, 2013. Green Paper on the Insurance of Natural and Man-made Disasters. COM/2013/0213 final.

[5] De Groeve, T., Poljansek, K. \& Ehrlich, D., Recording Disaster Losses. Recommendations for a European Approach, JRC Scientific and Policy Report. Report EUR 26111 En, 2013.

[6] Wirtz, A., Kron, W., Low, P. \& Steuer M., The need for data: natural disasters and challenges of database management, Nat Hazards, 70, pp. 135-157, 2014.

[7] Guha-Sapir D. \& Below R., The quality and accuracy of disaster data. A comparative analyses of three global data sets. ProVention Consortium (World Bank), 2002.

[8] Llsat et al., Towards a database on societal impact of Mediterranean floods within the framework of the HYMEX project, Nat. Hazards Earth Syst. Sci, 13, pp. 1337-1350, 2013.

[9] Mysiak, J., Testella, F., Bonaiuto, M., Carrus, G., De Dominicis, S., Ganucci Cancellieri, U., Firus, K. \& Grifoni, P., Flood risk management in Italy: challenges and opportunities for the implementation of the EU Floods Directive (2007/60/EC), Nat. Hazards Earth Syst. Sci., 13, pp. 2883-2890, 2013.

[10] Wergerdt, J. \& Mark, S.S., Post-Nargis Needs assessment and monitoring. ASEAN's Pioneering Response, Final report, Asean Secretariat, 2010.

[11] Queensland Reconstruction Authority, Australia, http://qldreconstruction.org.au/about/darmsys

[12] Jonkman, S.N., Bočkarjova, M., Kok, M. \& Bernardini P., Integrated hydrodynamic and economic modelling of flood damage in the Netherlands, Ecological Economics, 66, pp. 77-90, 2008.

[13] Servizio Protezione Civile - Regione Umbria. Evento alluvionale 11-14 Novembre 2012: Rapporto di evento. Available on line at: www.cfumbria.it

[14] Molinari, D., Menoni, S. , Aronica, G.T., Ballio, F., Berni, N., Pandolfo, C., Stelluti, M., Minucci, G., Ex-post damage assessment: an Italian experience, Nat. Hazards Earth Syst. Sci (accepted for publication). 